THIS IS AN AUTHOR-CREATED, UN-COPYEDITED VERSION OF AN ARTICLE ACCEPTED FOR PUBLICATION IN THE ASTROPHYSICAL JOURNAL LETTERS. IOP PUBLISHING LTD IS NOT RESPONSIBLE FOR ANY ERRORS OR OMISSIONS IN THIS VERSION OF THE MANUSCRIPT OR ANY VERSION DERIVED FROM IT. THE DEFINITIVE PUBLISHER AUTHENTICATED VERSION IS AVAILABLE ONLINE AT 10.1088/0004-637X/111/L1.

Preprint typeset using LTEX style emulateapj v. 08/13/06

\title{
XMM-NEWTON DISCOVERY OF 2.6 S PULSATIONS IN THE SOFT GAMMA-RAY REPEATER SGR 1627-41*
}

\author{
P. Esposito ${ }^{1,2}$, A. Tiengo ${ }^{1}$, S. Mereghetti ${ }^{1}$, G. L. Israel ${ }^{3}$, A. De LuCA ${ }^{1,4}$, D. Götz ${ }^{5}$, N. ReA $^{6}$, R. Turolla $^{7,8}$, And S. Zane $^{8}$ \\ ${ }^{1}$ INAF/Istituto di Astrofisica Spaziale e Fisica Cosmica - Milano, via E. Bassini 15, 20133 Milano, Italy; paoloesp@ iasf-milano.inaf.it \\ ${ }^{2}$ INFN - Istituto Nazionale di Fisica Nucleare, Sezione di Pavia, via A. Bassi 6, 27100 Pavia, Italy \\ ${ }^{3}$ INAF/Osservatorio Astronomico di Roma, via Frascati 33, 00040 Monteporzio Catone, Italy \\ ${ }^{4}$ IUSS - Istituto Universitario di Studi Superiori, viale Lungo Ticino Sforza, 56, 27100 Pavia, Italy \\ ${ }^{5}$ CEA Saclay, DSM/Irfu/Service d'Astrophysique, Orme des Merisiers, Bât. 709, 91191 Gif sur Yvette, France \\ ${ }^{6}$ Astronomical Institute “Anton Pannekoek," University of Amsterdam, Kruislaan 403, 1098 SJ Amsterdam, The Netherlands \\ ${ }^{7}$ Dipartimento di Fisica, Università degli Studi di Padova, via F. Marzolo 8, 35131 Padova, Italy and \\ ${ }^{8}$ Mullard Space Science Laboratory, University College London, Holmbury St. Mary, Dorking, Surrey RH5 6NT, UK \\ Received 2008 October 22; Accepted 2008 November 24
}

\begin{abstract}
After nearly a decade of quiescence, the soft gamma-ray repeater SGR 1627-41 reactivated on 2008 May 28 with a bursting episode followed by a slowly decaying enhancement of its persistent emission. To search for the still unknown spin period of this SGR taking advantage of its high flux state, we performed on 2008 September $27-28$ a 120 ks long X-ray observation with the $X M M$-Newton satellite. Pulsations with $P=2.594578(6) \mathrm{s}$ were detected at a higher than $6 \sigma$ confidence level, with a double-peaked pulse profile. The pulsed fraction in the $2-12 \mathrm{keV}$ range is $19 \% \pm 3 \%$ and $24 \% \pm 3 \%$ for the fundamental and the second harmonic, respectively. The observed 2-10 keV flux is $3.4 \times 10^{-13} \mathrm{erg} \mathrm{cm}^{-2} \mathrm{~s}^{-1}$, still a factor of $\sim 5$ above the quiescent pre-burstactivation level, and the spectrum is well fitted by an absorbed power law plus blackbody model (photon index $\Gamma \simeq 0.6$, blackbody temperature $k T \simeq 0.5 \mathrm{keV}$, and absorption $N_{\mathrm{H}} \approx 1.2 \times 10^{23} \mathrm{~cm}^{-2}$ ). We also detected a shell of diffuse soft X-ray emission which is likely associated with the young supernova remnant G337.0-0.1.
\end{abstract}

Subject headings: ISM: individual (G337.0-0.1) — stars: neutron — supernova remnants — X-rays: individual (SGR 1627-41) - X-rays: stars

\section{INTRODUCTION}

Two classes of young isolated neutron stars, the anomalous X-ray pulsars (AXPs) and the soft gamma-ray repeaters (SGRs) are currently believed to host magnetars, i.e. isolated neutron stars endowed with ultra-strong magnetic fields, $B \sim 10^{14}-10^{15} \mathrm{G}$ on the surface and one (or more) order of magnitude higher in the interior (Duncan \& Thompson 1992; Thompson \& Duncan 1993, 1995). SGRs and AXPs share a number of properties, including rotation periods in the 2 $12 \mathrm{~s}$ range, large period derivatives $\left(\dot{P} \sim 10^{-13}-10^{-9} \mathrm{~s} \mathrm{~s}^{-1}\right)$, inferred surface magnetic dipole field strength $B \gtrsim 10^{14} \mathrm{G}$, large and variable X-ray luminosities (exceeding that available from the braking of their rotation), and the emission of short bursts (see Woods \& Thompson 2006 and Mereghetti 2008 for recent reviews).

The five known Local Group SGRs were discovered as sources of short $(<1 \mathrm{~s})$ and intense $\left(10^{41}-10^{43} \mathrm{erg} \mathrm{s}^{-1}\right)$ bursts of gamma rays that they emit during sporadic periods of activity interrupted by long stretches of quiescence. Once localized through their bursts and flares, all of them were found to be persistent X-ray emitters, with luminosities of about $10^{33}$ $10^{36} \mathrm{erg} \mathrm{s}^{-1}$. In four SGRs, SGR 1627-41 being the exception, pulsations were detected with periods from 5 to $8 \mathrm{~s}$ and period derivatives of $10^{-11}-10^{-9} \mathrm{~s} \mathrm{~s}^{-1}$.

SGR 1627-41 was discovered in 1998 June by the Compton Gamma Ray Observatory thanks to the intense bursts it emitted at that time, about 100 in six weeks (Woods et al. 1999). Soon after the discovery of the bursts, its persistent X-

\footnotetext{
* Based on observations obtained with XMM-Newton, an ESA science mission with instruments and contributions directly funded by ESA Member States and NASA.
}

ray emission was detected by BeppoSAX at a flux level of $\sim$ $7 \times 10^{-12} \mathrm{erg} \mathrm{cm}^{-2} \mathrm{~s}^{-1}$ (unabsorbed, 2-10 keV), corresponding to a luminosity of $\sim 10^{35} \mathrm{erg} \mathrm{s}^{-1}$ for a source distance $d=11 \mathrm{kpc}$ (Corbel et al. 1999). This led to the possible association of the SGR with the young supernova remnant (SNR) G337.0-0.1 in the CTB 33 complex (Woods et al. 1999; Hurley et al. 1999). In the following 10 years no further bursting activity was reported while the persistent emission steadily decayed to about $10^{33} \mathrm{erg} \mathrm{s}^{-1}$ (for $d=11 \mathrm{kpc}$; Corbel et al. 1999), the lowest value ever observed from an SGR. At the same time the spectrum softened (Kouveliotou et al. 2003; Mereghetti et al. 2006).

The long-term fading of SGR 1627-41 was suddenly interrupted by its burst reactivation in 2008 May. This episode was associated with a temporary enhancement of the persistent $\mathrm{X}$-ray flux and a marked spectral hardening (Esposito et al. 2008).

The detection of SGR-like bursts from SGR 1627-41 made it a bona fide member of the SGR class. However, two strong pieces of evidence in favor of this identification, the measure of the source period and period derivative, were still missing. ${ }^{9}$ In order to search for pulsations taking advantage of the enhanced flux after the reactivation, we requested a Target of Opportunity XMM-Newton observation, that was performed as soon as the satellite visibility constraints allowed it.

\section{OBSERVATION, DATA ANALYSIS, AND RESULTS}

Our deep XMM-Newton observation of SGR 1627-41 started on 2008 September 25 at 00:51:02 UT and lasted about

\footnotetext{
${ }^{9}$ Woods et al. (1999) reported a candidate periodicity of marginal significance at $6.41 \mathrm{~s}$, but the signal was not confirmed by subsequent observations.
} 


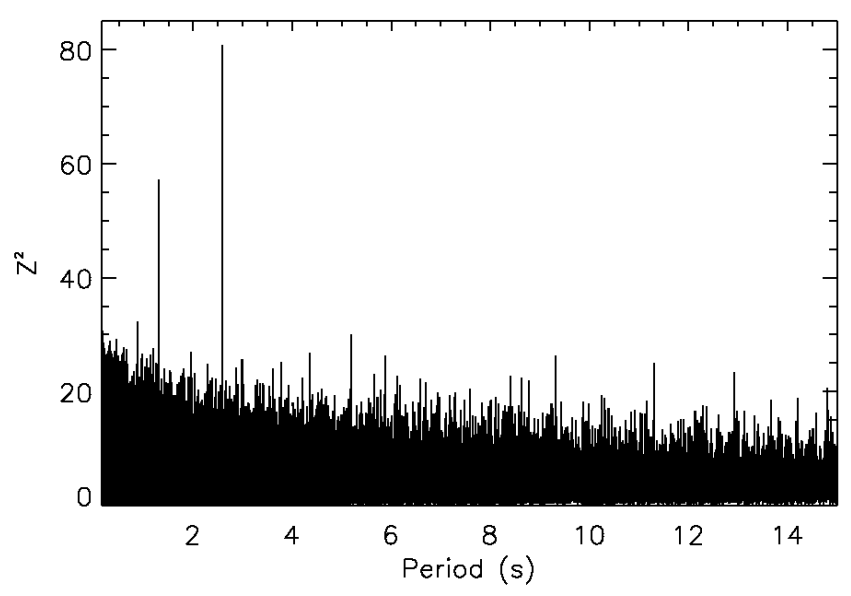

FIG. 1. $-Z_{2}^{2}$ periodogram computed for the pn data of SGR 1627-41. The $2.594578 \mathrm{~s}$ pulsed signal and its first harmonic stand out well above the noise level.

$120 \mathrm{ks}$, occupying the whole satellite revolution number 1611 . The data were collected with the EPIC instrument, which consists of two MOS (Turner et al. 2001) and one pn (Strüder et al. 2001) CCD cameras sensitive to photons with energies between 0.1 and $15 \mathrm{keV}$. The EPIC pn was operated in full frame mode (time resolution $73 \mathrm{~ms}$ ) while both MOS units were in small window mode (time resolution $0.3 \mathrm{~s}$ ). All the detectors mounted the thick optical blocking filter.

The raw observation data files (ODF) were processed with the XMM-Newton Science Analysis Software ${ }^{10}$ (SAS version 8.0.0) and the calibration files released in 2007 August using standard pipeline tasks (epproc and emproc for the pn and MOS, respectively). The source photons for the timing and spectral analysis were accumulated from a circular region (30 arcsec radius) centered on SGR 1627-41. The background events were extracted from source-free regions of the same chip as the source. We selected events with pattern $0-4$ and pattern $0-12$ for the pn and the MOS, respectively. For the timing analysis, the photon arrival times were converted to the solar system barycenter using the SAS task barycen. Photons having energies below $2 \mathrm{keV}$ and above $12 \mathrm{keV}$ were ignored, owing to the very few counts from SGR 1627-41. A total of about $2650 \pm 60$ counts above the background were collected from SGR $1627-41$ by the pn detector in the $2-12 \mathrm{keV}$ range, $760 \pm 40$ by the MOS 1 detector, and $900 \pm 40$ by the MOS 2 detector.

\subsection{Timing analysis}

For the timing analysis we started with the high timeresolution pn data. We searched for pulsations in the 0.15 $15 \mathrm{~s}$ range ${ }^{11}$ using the $Z_{n}^{2}$ test (Buccheri et al. 1983), with the number of harmonics $n$ being varied from 1 to 5 . The most significant peak occurred at about $2.6 \mathrm{~s}$ for $n=2$ (see Figure 1). Taking into account the number of searched harmonics and periods $(1,647,257)$, the $Z_{2}^{2}$ value of 80.72 for this peak corresponds to a chance probability of $\sim 9 \times 10^{-10}$ (that is a $>6 \sigma$ detection)

We refined the period measurement by folding the pn plus MOS data at the best period inferred through the $Z_{2}^{2}$ test and studying the phase evolution by means of a phase-fitting tech-

\footnotetext{
${ }^{10}$ See http://xmm.esac.esa.int/external/xmm_data_analysis/

${ }^{11}$ The frame time of the pn in full frame mode implies a Nyquist limit of about $146.6 \mathrm{~ms}$.
}

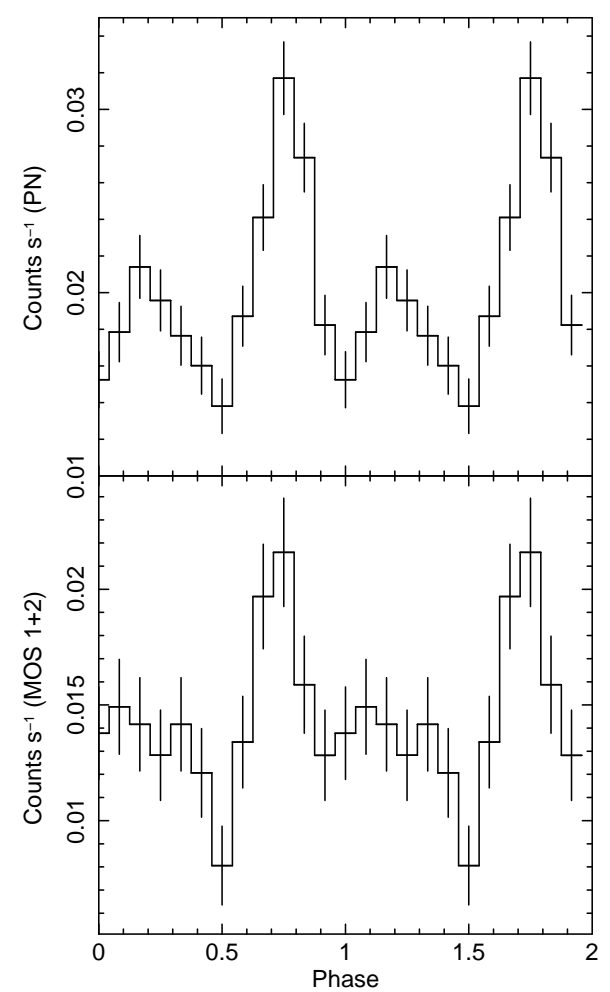

FIG. 2.- Epoch-folded pulse profile of SGR 1627-41 (2-12 keV) for the pn (top) and MOS data (bottom). The arrival time of the pulse minima (arbitrarely adopted as phase 0) was 54734.0000000 (4) MJD (TDB).

nique (see Dall'Osso et al. 2003 for more details). The resulting best-fit period was $P=2.594578(6)$ s (epoch 54734.0 MJD; hereafter all uncertainties are at $1 \sigma$ confidence level, unless otherwise specified). The current data set a $3 \sigma$ upper limit on the period derivative of $|\dot{P}|<6 \times 10^{-10} \mathrm{~s} \mathrm{~s}^{-1}$.

The background-subtracted lightcurves folded at the best period are shown in Figure 2. The pulse profile is double peaked, with a pulsed fraction (semiamplitude of sinusoidal modulation divided by the mean source count rate; see Israel $\&$ Stella 1996 for more details) of $19 \% \pm 3 \%$ and $24 \% \pm 3 \%$ for the fundamental and the second harmonic, respectively (the above values correspond to $16 \% \pm 4 \%$ in terms of root mean square, consistently with the $10 \%$ upper limit reported in Woods \& Thompson 2006). Within the statistical uncertainties the pulsed fraction is energy independent and no significant pulse shape variations as a function of energy were found by dividing the counts in soft and hard energy intervals. To assess this, we compared the folded lightcurves in various energy ranges using a two-dimensional KolmogorovSmirnov test (Peacock 1983; Fasano \& Franceschini 1987). The results show that the probability that they come from the same underlying distribution is always larger than $30 \%$.

Knowing the spin period, we went back to archival data and looked for pulsations with enhanced sensitivity. However, our searches in the previous XMM-Newton observations, as well as in BeppoSAX, ASCA and RossiXTE data, ${ }^{12}$ were inconclusive. The most constraining limits on the pulsed fraction were obtained from BeppoSAX (1998 August) and ASCA (1999 February) archival observations of SGR 1627-41. A Fourier analysis of the background-subtracted light curves was performed by means of the method described in Israel \& Stella

\footnotetext{
${ }^{12}$ Public available Chandra data of SGR 1627-41 could not be used owing to their inadequate frame times.
} 
TABLE 1

SPECTRAL RESULTS FOR SGR 1627-41.

\begin{tabular}{lccc}
\hline \hline \multicolumn{1}{c}{ Parameter } & \multicolumn{3}{c}{ Value } \\
\cline { 2 - 4 } & $\mathrm{BB}$ & $\mathrm{PL}$ & PL+BB \\
\hline$N_{\mathrm{H}}\left(10^{22} \mathrm{~cm}^{-2}\right)$ & $1.7 \pm 0.4$ & $5.2_{-0.5}^{+0.6}$ & $12 \pm 2$ \\
$\Gamma$ & $\ldots$ & $0.94_{-0.08}^{+0.06}$ & $0.6_{-0.2}^{+0.1}$ \\
$k T(\mathrm{keV})$ & $2.5_{-0.1}^{+0.2}$ & $\ldots$ & $0.50_{-0.06}^{+0.04}$ \\
$R(\mathrm{~km})$ & $0.041 \pm 0.003$ & $\ldots$ & $1.2 \pm 0.1$ \\
$\chi_{r}^{2} / \mathrm{dof}$ & $1.38 / 152$ & $1.19 / 152$ & $1.07 / 150$ \\
Flux $^{\mathrm{a}}\left(10^{-13} \mathrm{erg} \mathrm{cm}^{-2} \mathrm{~s}^{-1}\right)$ & 3.3 & 3.3 & 3.4 \\
\hline
\end{tabular}

NotE. - The abundances used are those of Anders \& Grevesse (1989) and photoelectric absorption cross-sections from BalucinskaChurch \& McCammon (1992). Blackbody temperature and radius are calculated at infinity and assuming a distance to the source of $11 \mathrm{kpc}$ (Corbel et al. 1999).

${ }^{\mathrm{a}}$ Observed flux in the $2-10 \mathrm{keV}$ range.

(1996) and no significant periodicity was found in the period interval 2.4-2.8 s (identified by considering the $3 \sigma$ upper limit on $\dot{P}$ inferred above). The $3 \sigma$ upper limits on the pulsed fraction (as defined above), computed according to Vaughan et al. (1994), are about $63 \%$ and $51 \%$ for the BeppoSAX and $A S C A$ data sets, respectively. ${ }^{13}$

\subsection{Spectral analysis}

For the spectral fitting (with XSPEC version 12.4; Arnaud 1996), data were grouped so as to have at least 30 counts per energy bin. The ancillary response files and the spectral redistribution matrices were generated with the SAS tasks arfgen and rmfgen, respectively. We jointly fitted the spectra by pn, MOS 1, and MOS 2 to blackbody, power law, and blackbody plus power law models, all corrected for interstellar absorption (see Table 1 for the best-fit model parameters).

The data disfavor the blackbody model $\left(\chi_{r}^{2}=1.38\right.$ for 152 degrees of freedom (dof)) and also the power-law fit yields a relatively high $\chi^{2}$ value $\left(\chi_{r}^{2}=1.19,152\right.$ dof $)$ with structured residuals (see Figure 3 ). A better fit is obtained by a power law plus blackbody model $\left(\chi_{r}^{2}=1.07,150\right.$ dof $)$. The best-fit parameters are photon index $\Gamma \simeq 0.6$, blackbody temperature $k T \simeq 0.5 \mathrm{keV}$, and absorption $N_{\mathrm{H}} \approx 1.2 \times 10^{23} \mathrm{~cm}^{-2}$. For a distance of $11 \mathrm{kpc}$ (Corbel et al. 1999) this corresponds to a luminosity of $\sim 10^{34} \mathrm{erg} \mathrm{s}^{-1}$ (2-10 keV, unabsorbed).

We compared the current spectral shape of SGR 1627-41 with those inferred from the latest observations by means of simultaneous fits. Apart from an overall normalization factor (to account for the different luminosities), the current spectrum is consistent with that observed by Swift/XRT during the onset of the 2008 May outburst, while it is significantly harder than that observed by XMM-Newton before the outburst, in 2008 February (Esposito et al. 2008).

\subsection{Imaging analysis}

The energy-coded image of the field around SGR 1627-41 (Figure 4) shows that the source is embedded in a complex region of diffuse emission. A shell-shaped structure $(\sim 2$ arcmin diameter) with a softer spectrum than that of the SGR, as well as a bright spot of remarkably hard diffuse emission $(\sim 2$ arcmin to the southwest of SGR 1627-41) are apparent in Figure 4, where red and blue correspond to soft and hard X-ray

\footnotetext{
13 These limits do not take into account the contribute from the diffuse structures described in Section 2.3, that are not resolved in the BeppoSAX and $A S C A$ data.
}

photons, respectively. The combination of large absorption, source confusion and small photon statistics makes spectral analysis of such diffuse structures rather difficult. Selecting photons from $\mathrm{a} \sim 100^{\prime \prime} \times 50^{\prime \prime}$ rectangular region located between SGR 1627-41 and the hard spot, we observe the emission of the very faint diffuse shell to peak between 2 and 3 $\mathrm{keV}$. Using Chandra imaging data of the field, we estimated the contribution of unresolved point-like sources to the diffuse shell to be of order $30 \%$ in the $1.7-3.1 \mathrm{keV}$ energy range. The brightest point sources in the region are also resolved in the EPIC data below $1.7 \mathrm{keV}$, where the diffuse emission is not seen. The spectrum of the shell is adequately described $\left(\chi_{r}^{2}=1.13,106\right.$ dof $)$ by an absorbed $\left(N_{\mathrm{H}} \sim 8 \times 10^{22} \mathrm{~cm}^{-2}\right)$ hot plasma model (pshock in XSPEC, $k T \sim 1 \mathrm{keV}$ ), with clear indications for emission lines from $\mathrm{S}$ (at 2.46 and $3.10 \mathrm{keV}$ ) and possibly from $\mathrm{Fe}$ (at $6.4 \mathrm{keV}$ ) and is therefore consistent with emission from a very absorbed SNR. The net (point source subtracted) surface brightness of the structure is of $\sim 10^{-14}$ erg $\mathrm{cm}^{-2} \mathrm{~s}^{-1}$ arcmin $^{-2}$ in the $1.7-3.1 \mathrm{keV}$ band.

The spectrum of the second, harder, diffuse structure was extracted using a 20 arcsec radius circle around the apparent centroid of the feature. The source emission emerges from background above $\sim 2 \mathrm{keV}$ and peaks around $5 \mathrm{keV}$. Significant signal is detected up to $12 \mathrm{keV}$. A thermal plasma model (mekal in XSPEC, abundance parameter left free) yields an acceptable description of the data $\left(\chi_{r}^{2}=1.27,53 \mathrm{dof}\right)$, although the absorbing column and the temperature are not well constrained $\left(N_{\mathrm{H}}>1.3 \times 10^{23} \mathrm{~cm}^{-2}, k T>13 \mathrm{keV}\right.$ at $90 \%$ confidence level for a single parameter). The possible presence of an emission feature at $\sim 6.2 \mathrm{keV}$ makes the fit improve after freeing the redshift parameter $\left(\chi_{r}^{2}=1.08,52\right.$ dof; bestfit $z \sim 0.1$, pointing to Fe emission at $6.9 \mathrm{keV}$ in the source frame). The $2-10 \mathrm{keV}$ flux of the source within the aperture is of $\sim 1.2 \times 10^{-13} \mathrm{erg} \mathrm{cm}^{-2} \mathrm{~s}^{-1}$. A possible interpretation is that this source is a background cluster of galaxies. We note that a simple absorbed $\left(N_{\mathrm{H}} \sim 1.4 \times 10^{23} \mathrm{~cm}^{-2}\right)$ power law $(\Gamma \sim 1.6)$ also yields an acceptable fit ( $\left.\chi_{r}^{2}=1.27,53 \mathrm{dof}\right)$. Thus, the source could also be a pulsar wind nebula, unrelated to SGR 1627-41.

\section{DISCUSSION AND CONCLUSIONS}

We have discovered pulsations with period of $2.6 \mathrm{~s}$ in SGR 1627-41, which was the only known magnetar with no spin period yet measured. The pulse shape is double peaked, with pulsed fractions of $\sim 19 \%$ and $24 \%$ in the two harmonics. No dependence of the pulse profile on the energy band has been found. Among the known magnetars, only 1E 1547.0-5408 has a shorter period (2.07 s; Camilo et al. 2007) than that of SGR 1627-41. We searched for a similar periodicity in all available archival data with sufficient time resolution, but no pulsation was found. Relying only on the last $X M M$-Newton observation we could set an upper limit on the period derivative, $|\dot{P}|<6 \times 10^{-10} \mathrm{~s} \mathrm{~s}^{-1}$ which is not particularly constraining for a magnetar, and yields $B \lesssim 1.2 \times 10^{15} \mathrm{G}$. Based on the peak luminosities of bursts observed from SGR 1627-41, its dipole magnetic field was estimated to be $B \gtrsim 1.8 \times 10^{14} \mathrm{G}$ (Woods et al. 1999 ; Esposito et al. 2008). This limit can be used to infer a period derivative $\dot{P} \gtrsim 1.2 \times 10^{-11} \mathrm{~s} \mathrm{~s}^{-1}$, a spin-down luminosity $\dot{E} \gtrsim 8 \times 10^{33}$ $\mathrm{erg} \mathrm{s}^{-1}$, and a characteristic age $\tau_{c}=P /(2 \dot{P}) \lesssim 3.4 \mathrm{kyr}$. Overall, and lacking any information on the pulsation period and profile in the past, we can only conclude that the timing properties of SGR 1627-41 reported here appear quite typical 


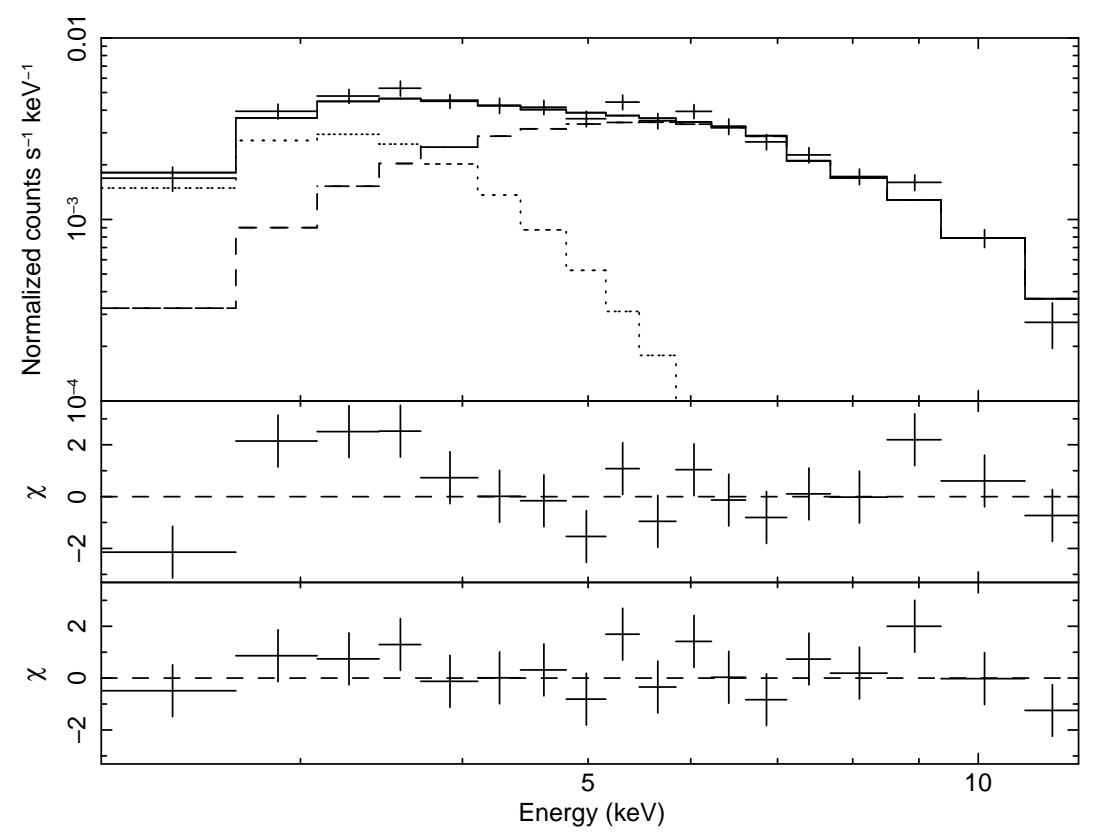

FIG. 3.- EPIC/pn spectrum of SGR 1627-41. Top: data and best-fit power law plus blackbody model (solid line); the dotted (blackbody) and dashed line (power law) show the individual components. Middle: residuals from the power-law best-fit model in units of standard deviation. Bottom: residuals from the power law plus blackbody best-fit model in units of standard deviation. The data have been rebinned in the plot to better visualize the trend in the spectral residuals.

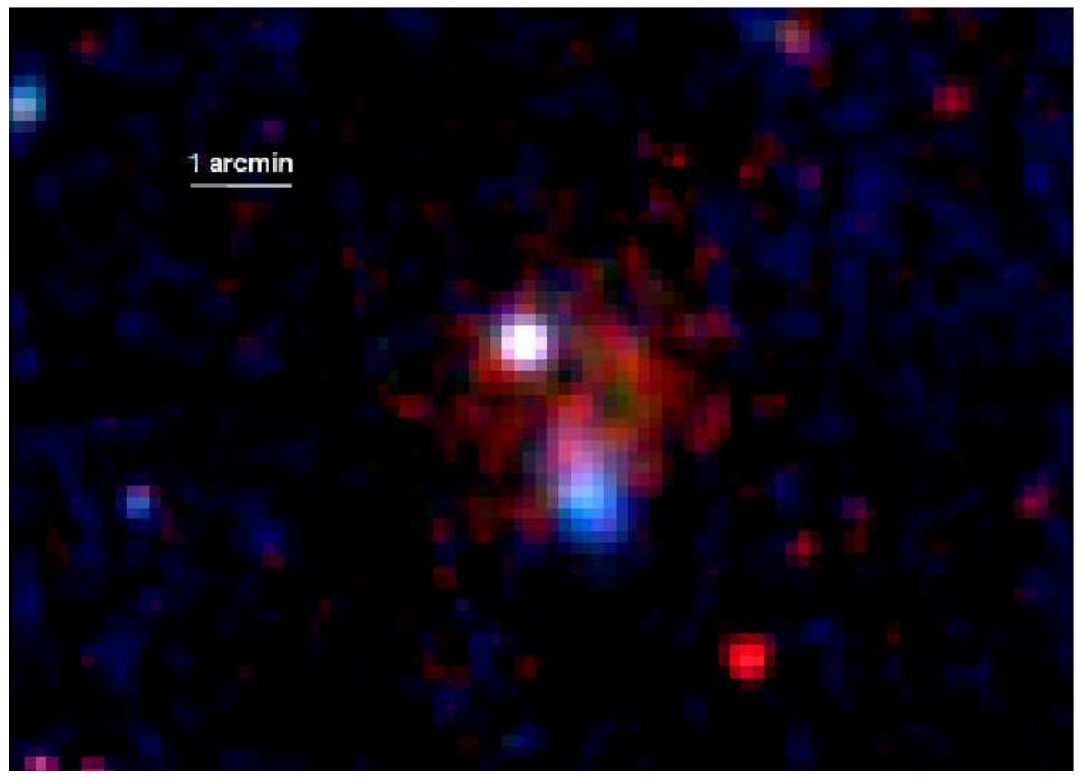

FIG. 4.- EPIC/pn image of the field of SGR 1627-41 $\left(11^{\prime} \times 7^{\prime}\right)$. Data from the long 2008 September observation are combined with previous XMM-Newton data, totaling to $147.8 \mathrm{ks}$ exposure time. Photon energy is color-coded: red corresponds to the $1.7-3.1 \mathrm{keV}$ energy range, green to $3.1-5.0 \mathrm{keV}$, blue to $5.0-8.0$ $\mathrm{keV}$. North is up, east is left. SGR 1627-41, in white, is the brightest source in the field. The image unveils diffuse X-ray emission from SNR G337.0-0.1, prominent in the softest energy range. A patch of hard diffuse emission is also apparent, southwest of the SNR, possibly due to a background, very absorbed cluster of galaxies.

of a member of the SGR class.

Our observation caught SGR 1627-41 at a flux of $3.4 \times 10^{-13} \mathrm{erg} \mathrm{cm}^{-2} \mathrm{~s}^{-1}$, the highest seen with XMM-Newton and a factor of $\sim 5$ above the level preceding its 2008 May burst activation (see Figure 1 of Esposito et al. 2008 for a complete flux history of the source). Its spectrum was well fitted in earlier data by a single-component model, either a heavily absorbed power law or a blackbody (Mereghetti et al. 2006). The higher-quality spectrum of the new data cannot be adequately fitted by these simple models, while it is consistent with a power law plus blackbody model, and it is significantly harder than in any observation of SGR 1627-41 taken before 2008 May (Kouveliotou et al. 2003; Mereghetti et al. 2006). The presence of a soft thermal component and the spectral hardening as the source moves from a quiescent to a (burst) active phase makes SGR 1627-41 akin to other SGRs and AXPs (e.g. Mereghetti et al. 2005; Rea et al. 2005). This latter behavior can be interpreted in the framework of the "twisted-magnetosphere model" as due to a progressive growth of the shear in the magnetosphere as magnetic helicity is transferred from the internal to the external field (Thompson et al. 2002; Fernández \& Thompson 
2007; Nobili et al. 2008).

SGR 1627-41 has been proposed to be associated (Woods et al. 1999; Hurley et al. 1999, but see also Gaensler et al. 2001) to the radio SNR G337.0-0.1 (Frail et al. 1996; Whiteoak \& Green 1996). The shell of diffuse soft radiation (Section 2.3), consistent with emission from a young SNR at the distance of SGR 1627-41, is likely to be the first detection of this SNR in X-rays. Due to the complexity of the region, the extension of the SNR at radio frequencies is debated: Sarma et al. (1997) claimed a diameter of $\sim 95$ arcsec with SGR 1627-41 outside the shell, while Brogan et al. (2000) proposed a different morphology extending the SNR size towards the southwest and encompassing the SGR. The position and extension of the diffuse X-ray emission are consistent with the latter hypothesis, supporting the possible association between SGR 1627-41 and G337.0-0.1.

We thank Norbert Schartel and the staff of the XMMNewton Science Operation Center for performing this Target of Opportunity observation. We thank Pat Romano, Neil Gehrels and Rhaana Starling for their help in planning the observation. We also thank Fabio Gastaldello and Marta Burgay for their helpful advice on the analysis of the field of SGR 1627-41. The Italian authors acknowledge the partial support from ASI (ASI/INAF contracts I/088/06/0 and AAE TH-058). D.G. acknowledges the CNES for financial support. N.R. is supported by an NWO Veni Fellowship. S.Z. acknowledges support from STFC.

\section{REFERENCES}

Anders, E. \& Grevesse, N. 1989, Geochim. Cosmochim. Acta, 53, 197

Arnaud, K. A. 1996, in ASP Conf. Ser. 101, Astronomical Data Analysis Software and Systems V, ed. G. Jacoby \& J. Barnes (San Francisco, CA ASP), 17

Balucinska-Church, M. \& McCammon, D. 1992, ApJ, 400, 699

Brogan, C. L., Frail, D. A., Goss, W. M., \& Troland, T. H. 2000, ApJ, 537, 875

Buccheri, R., et al. 1983, A\&A, 128, 245

Camilo, F., Ransom, S. M., Halpern, J. P., \& Reynolds, J. 2007, ApJ, 666, L93

Corbel, S., Chapuis, C., Dame, T. M., \& Durouchoux, P. 1999, ApJ, 526, L29

Dall'Osso, S., Israel, G. L., Stella, L., Possenti, A., \& Perozzi, E. 2003, ApJ, 599,485

Duncan, R. C. \& Thompson, C. 1992, ApJ, 392, L9

Esposito, P., et al. 2008, MNRAS, 390, L34

Fasano, G. \& Franceschini, A. 1987, MNRAS, 225, 155

Fernández, R. \& Thompson, C. 2007, ApJ, 660, 615

Frail, D. A., Goss, W. M., Reynoso, E. M., Giacani, E. B., Green, A. J., \& Otrupcek, R. 1996, AJ, 111, 1651

Gaensler, B. M., Slane, P. O., Gotthelf, E. V., \& Vasisht, G. 2001, ApJ, 559, 963

Hurley, K., Kouveliotou, C., Woods, P., Mazets, E., Golenetskii, S., Frederiks, D. D., Cline, T., \& van Paradijs, J. 1999, ApJ, 519, L143
Israel, G. L. \& Stella, L. 1996, ApJ, 468, 369

Kouveliotou, C., et al. 2003, ApJ, 596, L79

Mereghetti, S. 2008, A\&A Rev., 15, 225

Mereghetti, S., et al. 2006, A\&A, 450, 759

Mereghetti, S., et al. 2005, ApJ, 628, 938

Nobili, L., Turolla, R., \& Zane, S. 2008, MNRAS, 386, 1527

Peacock, J. A. 1983, MNRAS, 202, 615

Rea, N., Tiengo, A., Mereghetti, S., Israel, G. L., Zane, S., Turolla, R., \& Stella, L. 2005, ApJ, 627, L133

Sarma, A. P., Goss, W. M., Green, A. J., \& Frail, D. A. 1997, ApJ, 483, 335

Strüder, L., et al. 2001, A\&A, 365, L18

Thompson, C. \& Duncan, R. C. 1993, ApJ, 408, 194

-. 1995, MNRAS, 275, 255

Thompson, C., Lyutikov, M., \& Kulkarni, S. R. 2002, ApJ, 574, 332

Turner, M. J. L., et al. 2001, A\&A, 365, L27

Vaughan, B. A., et al. 1994, ApJ, 435, 362

Whiteoak, J. B. Z. \& Green, A. J. 1996, A\&AS, 118, 329

Woods, P. M., et al. 1999, ApJ, 519, L139

Woods, P. M. \& Thompson, C. 2006, in Compact Stellar X-ray Sources, ed W. H. G. Levin and M. van der Klis (Cambridge: Cambridge Univ. Press), 547 